\title{
Therapeutic activity of superoxide dismutase-containing enzymosomes on rat liver ischaemia-reperfusion injury followed by magnetic resonance microscopy
}

\author{
Paulo Marcelino $^{\mathrm{a}}$, H. Susana Marinho ${ }^{\mathrm{b}}$, Maria Celeste Campos ${ }^{\mathrm{c}}$, Ana Rita Neves ${ }^{\mathrm{b}}$, Carla Real ${ }^{\mathrm{b}}$, \\ Filipa S. Fontes ${ }^{\mathrm{b}, \mathrm{d}}$, Alexandra Carvalho ${ }^{\mathrm{e}, \mathrm{f}}$, Gabriel Feio ${ }^{\mathrm{e}}$, M. Bárbara F. Martins ${ }^{\mathrm{d}}$, M. Luísa Corvo ${ }^{\mathrm{d}, *}$ \\ a CEDOC, Nova Medical School, Campo dos Mártires da Pátria, 130, 1169-056 Lisboa, Portugal \\ b Centro de Química e Bioquímica, Faculdade de Ciências, Universidade de Lisboa, Campo Grande, 1749-016 Lisboa, Portugal and Departamento de Química e \\ Bioquímica, Faculdade de Ciências, Universidade de Lisboa, 1749-016 Lisboa, Portugal \\ ${ }^{\mathrm{c}}$ Anatomia Patológica, Hospital Curry Cabral, Rua da Beneficência n. ${ }^{\circ}$ 8, 1069-166 Lisboa, Portugal \\ d Instituto de Investigação do Medicamento (iMed.ULisboa), Faculdade de Farmácia, Universidade de Lisboa, Avenida Professor Gama Pinto, 1649-003, Lisboa, Portugal \\ and Departamento de Farmácia Galénica e Tecnologia Farmacêutica, Faculdade de Farmácia, Universidade de Lisboa, Avenida Professor Gama Pinto, 1649-003 Lisboa, \\ Portugal \\ e CENIMAT-I3N-DCM, Faculdade de Ciências e Tecnologia da Universidade Nova de Lisboa, Campus da Caparica, 2829-515 Caparica, Portugal \\ ${ }^{\mathrm{f}}$ IEQUALTECS, Lda, R. Dr. Francisco Sá Carneiro, 36, 2500-065 S. Gregório CLD, Portugal
}

\section{A R T I C L E I N F O}

\section{Keywords:}

Superoxide dismutase enzymosomes

PEGylated liposomes

Antioxidant therapy

Liver ischaemia/reperfusion

MRM

Magnetic resonance microscopy

Targeting

Nanomedicine

Liver transplant

\begin{abstract}
A B S T R A C T
Liver ischaemia-reperfusion injury (IRI) may occur during hepatic surgery and is unavoidable in liver transplantation. Superoxide dismutase enzymosomes (SOD-enzymosomes), liposomes where SOD is at the liposomal surface expressing enzymatic activity in intact form without the need of liposomal disruption, were developed with the aim of having a better insight into its antioxidant therapeutic outcome in IRI. We also aimed at validating magnetic resonance microscopy (MRM) at $7 \mathrm{~T}$ as a tool to follow IRI. SOD-enzymosomes were characterized and tested in a rat ischaemia-reperfusion model and the therapeutic outcome was compared with conventional long circulating SOD liposomes and free SOD using biochemical liver injury biomarkers, histology and MRM. MRM results correlated with those obtained using classical biochemical biomarkers of liver injury and liver histology. Moreover, MRM images suggested that the therapeutic efficacy of both SOD liposomal formulations used was related to prevention of peripheral biliary ductular damage and disrupted vascular architecture. Therefore, MRM at $7 \mathrm{~T}$ is a useful technique to follow IRI. SOD-enzymosomes were more effective than conventional liposomes in reducing liver ischaemia-reperfusion injury and this may be due to a short therapeutic window.
\end{abstract}

\section{Introduction}

The interruption of blood flow to the liver is common during hepatic surgery and unavoidable in liver transplantation (LT). The lack of blood supply and consequent lack of oxygen and nutrients leads to cell damage through several mechanisms. This damage can be further exacerbated during liver revascularization (reperfusion), a clinical phenomenon known either as post-reperfusion syndrome or as ischaemiareperfusion (IR) injury (IRI).
Liver injury due to ischaemia-reperfusion can be divided into two major categories. The first is warm IRI, which is initiated by hepatocyte damage and which occurs when the blood flow is temporarily interrupted during transplantation, trauma and shock and elective liver surgery. The second is cold IRI which is initiated by damage to hepatic sinusoidal endothelial cells and microcirculation disruption occurs when organs are preserved for transplantation and is usually coupled with warm IRI during LT (Klune and Tsung, 2010; Zhai et al., 2013).

The hallmark of IRI is the local inflammatory innate immune

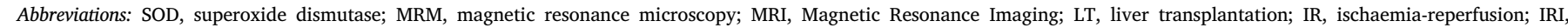

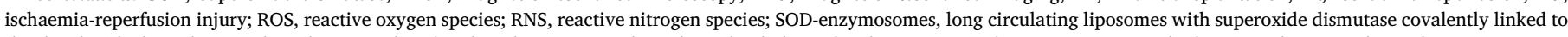
the distal end of 1,2-distearoyl-sn-glycero-3-phosphoethanolamine- $N$-[maleimide (polyethylene glycol)-2000]; SOD-liposomes, superoxide dismutase long circulating liposomes

* Corresponding author.

E-mail addresses: p.marcelino@netcabo.pt (P. Marcelino), smarinho@ciencias.ulisboa.pt (H.S. Marinho), celestecamposstar@gmail.com (M.C. Campos), anaritarneves@hotmail.com (A.R. Neves), csafonso@ciencias.ulisboa.pt (C. Real), filipasalavessafontes@gmail.com (F.S. Fontes), alexandra.carvalho@iequaltecs.pt (A. Carvalho), gf@fct.unl.pt (G. Feio), barbaramartins@ff.ulisboa.pt (M.B.F. Martins), lcorvo@ff.ulisboa.pt (M.L. Corvo). 
activation response that is triggered. In liver IRI there is initially an ischemic injury, which includes a series of molecular events occurring during hypoxia which may lead to cell death after depletion of cellular ATP. This response is directed at organ preservation and repair, and is genetically determined, and thus can vary from patient to patient. In the reperfusion phase of IRI, two distinctive phases can be singled out: the first phase, occurring in the initial $1-2 \mathrm{~h}$ of reperfusion, is characterized by a significantly impaired microcirculation which prolongs the period of hypoxia, with areas of the liver remaining ischemic after the onset of reperfusion (Serracino-Inglott et al., 2001). This in turn is followed by activation of immunocompetent cells, like resident liver macrophages (Kupffer cells) and generation of reactive oxygen species (ROS), such as superoxide radical $\left(\mathrm{O}_{2}{ }^{-}\right)$and hydrogen peroxide $\left(\mathrm{H}_{2} \mathrm{O}_{2}\right)$, and reactive nitrogen (RNS) species, such as nitric oxide ( $\mathrm{NO}$ ) and peroxynitrite $\left(\mathrm{ONOO}^{-}\right)$, leading to an initial stage of the inflammatory process and to oxidative stress. Activation of liver Kupffer cells is associated not only with increased rates of formation of ROS and RNS, but also with the release of proinflammatory cytokines (TNF- $\alpha$, IL$1 \beta$, IFN- $\gamma$, and others), chemokines (MIP-2, MCP-1) and adhesion molecules, which mediate signalling pathways ensuing the recruitment of inflammatory cells, such as neutrophils, and liver infiltration by neutrophils (Diesen and Kuo, 2011; Jaeschke, 2011; Klune and Tsung, 2010). The second phase, occurring 6-48 $\mathrm{h}$ after reperfusion, involves accumulation of neutrophils and hepatocellular injury (Jaeschke and Farhood, 2002; Klune and Tsung, 2010; Lutz et al., 2010).

High levels and/or inadequate removal of $\mathrm{O}_{2}{ }^{-}{ }^{-}$and $\mathrm{H}_{2} \mathrm{O}_{2}$ by antioxidant enzymes results in molecular damage and disruption of redox signalling i.e., oxidative stress, which may cause oxidative stressmediated diseases. $\mathrm{O}_{2}{ }^{-}-$main reactions in vivo are its dismutation, catalyzed by superoxide dismutases (SODs), to form $\mathrm{H}_{2} \mathrm{O}_{2}$ and oxygen and its reaction with nitric oxide to form peroxynitrite (Beckman and Koppenol, 1996). Superoxide dismutases started to be used as antioxidant therapy for inflammatory situations in the late 60s. Since then, SODs therapy has been shown to have a protective role, not just for inflammatory diseases, but also for other pathological situations where $\mathrm{O}_{2} \cdot-$ and endogenous SODs have a key role (Corvo et al., 2016). In fact, SODs have been used both in animal models and, also clinically, displaying positive therapeutic outcomes for rheumatoid arthritis, cancer, ischaemia-reperfusion injury, respiratory diseases, nervous tissue damage, infectious diseases and also for radiation side effects, such as fibrosis (Carillon et al., 2013).

Recently we began studies to establish both the therapeutic effectiveness of different liposomal formulations of bovine CuZn-SOD on animal models of arthritis and IRI, as well as to test a new contrast agent for Magnetic Resonance Imaging (MRI) with high spatial resolution (magnetic resonance microscopy, MRM) (Corvo et al., 2015; Martins et al., 2014). MRM has been suggested as a promising technique for medical imaging not only to diagnose acute ischaemia but also to follow up the complex chain of events that follows an acute ischaemia (Cheung et al., 2011; Martins et al., 2014). MRM can be used on live animals and when used for imaging of fixed specimens has advantages over conventional microscopy because it is non-destructive, can be 3D, and is inherently digital (Driehuys et al., 2008). Moreover, since the fixed samples do not have to be dehydrated for MRM, as it happens in conventional histology, MRM images display the natural distribution of water in tissues and organs and provide a detailed functional and anatomical picture thus allowing to characterize lesions not possible to visualize in other conditions (Driehuys et al., 2008). We developed long circulating liposomes with SOD covalently linked to the distal end of DSPE-PEG (SOD-enzymosomes) which when tested in an animal model of arthritis showed an enhanced therapeutic result. An animal model of liver IRI was used to make a preliminary assessment of the outcome of treatment with SOD-enzymosomes and with SOD-long circulating liposomes (SOD-liposomes). We found that treatment with SOD-enzymosomes, unlike SOD-liposomes or free SOD, decreased significantly alanine aminotransferase (AST) activity in serum, a biomarker of liver cell damage due to IRI. As previously demonstrated, both these nanosystems have long circulating capabilities and enhanced retention at inflamed sites/pathologies (Corvo et al., 1999, 2015; Martins et al., 2014). At $24 \mathrm{~h}$ post-injection, both formulations have similar biodistribution profiles, namely in the liver (Corvo et al., 2015). However, SOD-enzymosomes have a unique capability of expressing enzymatic activity in intact form without the need of liposomal disruption, thus enabling a faster therapeutic outcome when compared to SOD-liposomes. Our previous results suggested that a therapeutic approach to IRI using SOD-enzymosomes would be beneficial, decreasing liver cell damage due to ischaemia-reperfusion. Here we aimed at further characterizing the therapeutic effect of the administration of two liposomal formulations of SOD (SOD-long circulating liposomes) and SOD-enzymosomes in the final stage of liver ischaemia induced in rats by evaluating their effect on IRI. We also aimed at validating MRM as a tool to follow IRI by using known liver injury biochemical biomarkers and histological analyses.

\section{Materials and methods}

\subsection{Materials}

1,2-Distearoyl-sn-glycero-3-phosphoethanolamine- $N$-[maleimide (polyethylene glycol)-2000] (ammonium salt), (maleimide-PEG-PE) was purchased from Avanti Polar Lipids. Egg-phosphatidylcholine (PC) and 1,2-distearoyl-sn-glycero-3-phosphoethanolamine- $N$-[methoxy (polyethylene glycol)-2000] (PEG-PE) were obtained from Lipoid $\mathrm{GmbH}$. Bovine $\mathrm{Cu}, \mathrm{Zn}$-superoxide dismutase (SOD), dimethylformamide (DMF), $\mathrm{N}$-(2-hydroxyethyl) and Cholesterol (Chol), were obtained from Sigma. Ethylenediaminetetraacetic (EDTA) was from Merck. $N$-succinimidyl $S$-acethylthioacetate (SATA) was from Pierce. Imalgene $1000^{\circledR}$ (ketamine) was obtained from Merial Portuguesa Saúde Animal Lda, Rio de Mouro, Portugal and Domitor ${ }^{\circledR}$ (medetomidine) and Antisedam ${ }^{\circledR}$ (atipamezole) were obtained from Pfizer Animal Health, Pennsylvania, EUA.

\subsection{Animals}

For the therapeutic activity experiments male Wistar rats weighting 200-250 g were provided by Instituto Bento da Rocha Cabral, Lisbon, Portugal. Animals were fed with standard laboratory food and water ad libitum. All animal experiments were carried with the permission of the local animal ethical committee, and in accordance with the Declaration of Helsinki, EEC Directive (2010/63/UE) and Portuguese Law (DL 113/ 2013, Despacho $n^{\circ} 2880 / 2015$ ), and all following legislations for the humane care of animals in research.

\subsection{Methods}

\subsubsection{SOD formulations for therapeutic activity studies}

2.3.1.1. SOD-enzymosomes. SOD-enzymosomes were prepared as described in (Corvo et al., 2015). Briefly, PC:Chol:Maleimide-PEGPE:PEG-PE at the molar ratio of 68.25:30.5:0.75:0.5 and dispersed in $145 \mathrm{mM} \mathrm{NaCl} / 10 \mathrm{mM}$ citrate buffer, $\mathrm{pH} 6.0$ (20 $\mu \mathrm{mol}$ lipid/mL), dimensioned through a final polycarbonate membrane pore size of $0.05 \mu \mathrm{m}$. The conjugation was performed with a SATA:SOD molar ratio of $4: 1$ and a SOD concentration of $1 \mathrm{mg} / \mathrm{mL}$. The conjugated enzyme was separated from the enzymosome dispersion by ultracentrifugation, at $300,000 \times g$ for $120 \mathrm{~min}$ at $4{ }^{\circ} \mathrm{C}$ in a Beckman LM-80 ultracentrifuge. Finally, liposomes were dispersed in $0.145 \mathrm{M} \mathrm{NaCl} / 10 \mathrm{mM}$ citrate buffer pH 5.6.

2.3.1.2. SOD-liposomes. SOD-liposomes were prepared as described before (Corvo et al., 2002). Briefly, liposomes were prepared by the dehydration-rehydration method, followed by sequential extrusion through polycarbonate filters up to a final pore size of $0.05 \mu \mathrm{m}$. The 
lipid (PC:Chol:PEG-DSPE, 1.85:1:0.15 molar ratio) concentration was $32 \mu \mathrm{mol}$ lipid per $\mathrm{mL}$ hydration medium and the film was dispersed in a solution of SOD $(5 \mathrm{mg} / \mathrm{mL})$. Nonencapsulated enzyme was separated from the liposome dispersion by ultracentrifugation, at $300,000 \times g$ for $120 \mathrm{~min}$ at $4{ }^{\circ} \mathrm{C}$ in a Beckman LM-80 ultracentrifuge. Finally, liposomes were dispersed in $0.145 \mathrm{M} \mathrm{NaCl} / 10 \mathrm{mM}$ citrate buffer $\mathrm{pH}$ 5.6.

\subsubsection{Characterization of the SOD-enzymosomes and SOD-liposomes}

SOD content either when coupled or encapsulated to liposomes was determined according to Lowry et al. (1951) with prior disruption of liposomes with Triton X-100 and sodium dodecyl sulfate (Corvo et al., 2002; Wang and Smith, 1975). Phospholipids were quantified according to the method of Rouser et al. (1970).

The catalytic activity of SOD was measured according to Misra and Fridovich (1972), which is based on the ability of the enzyme to inhibit the autoxidation of epinephrine at $\mathrm{pH}$ 10.2. In the case of determination of liposome-encapsulated enzyme, the enzyme was first released from SOD-liposomes by the addition of $20 \%(\mathrm{v} / \mathrm{v})$ Triton X-100 (yielding a dispersion containing $10 \%(\mathrm{v} / \mathrm{v})$ Triton $\mathrm{X}-100)$. In the case of SODenzymosomes, when required, vesicles were also destroyed by the addition of $20 \%(\mathrm{v} / \mathrm{v})$ Triton X-100. In all activity measurements (either the enzyme solution or the SOD-enzymosome or SOD-liposome) suspensions were diluted to a concentration of $6 \mu \mathrm{g} / \mathrm{mL}$ [yielding a dispersion containing $0.3 \%$ Triton X-100 (v/v)]. The retention of enzyme activity was defined as:

Ret. Act. $=($ Final activity/Initial activity $)$

$\times 100(\%)$, taking the activity of native SOD as $100 \%$.

The hydrodynamic diameter (z-average) and polydispersity index (PdI) were measured in a Zetasizer Nano $S$ from Malvern and the Zeta potential in a Zetasizer Nano $\mathrm{Z}$ from Malvern.

\subsubsection{Liver ischaemia-reperfusion model}

Random groups of 5 animals (Wistar rats) were anaesthetized with $0.2 \mathrm{~mL}$ of a mixture 1:1 (v/v) of Imalgene $1000^{\circledR}$ (Merial Portuguesa Saúde Animal Lda, Rio de Mouro, Portugal) and Domitor ${ }^{\circledR}$ (Pfizer Animal Health, Pennsylvania, EUA). After anaesthetic induction, rats were weighed, an abdominal trichotomy was performed and they were placed on the work table. Afterwards, the abdomen was disinfected with an aseptic solution (Cutasept ${ }^{\circledR}$, Bode Chemie, Hamburg). A median laparotomy was performed and the liver hilum was exposed. After the identification of the vascular structures, they were joined with silk $2 / 0$ sutures. The suture was performed in the following manner: the posterior perivascular fat was debrided using mosquito forceps with curved tips and with the exposed tip of the mosquito, the silk suture was pulled; then, a plastic cylinder object with approximately $2 \mathrm{~cm}$ in length was placed and the suture knot was performed on top of this object (to avoid vascular lesion and haemorrhage, both while suturing and while cutting the silk). The same procedure was performed for the portal vein and liver ischaemia was considered from this moment on.

The surgical wound was protected and there was a $30 \mathrm{~min}$ wait after which the sutures were cut and liver irrigation was re-established. SOD therapeutic treatment, SOD, SOD-liposomes, SOD-enzymosomes were administered iv in the tail vein at a dose of $66 \mu \mathrm{g}$ SOD per rat a few minutes before the cut of sutures and re-establishment of liver irrigation. Each treatment group contained at least 5 rats. Antisedan ${ }^{\circledR}$ $(0.2 \mathrm{~mL})$ was administered to the animals for recovery from the anaesthesia. After $24 \mathrm{~h}$, animals were sacrificed. Blood was collected by cardiac puncture for the assay of liver ischaemia-reperfusion injury biomarkers. The liver was extracted, washed with $0.154 \mathrm{mM} \mathrm{KCl}$ and either stored at $-80^{\circ} \mathrm{C}$ or, when used for MRI and histological studies, kept in a $10 \%(\mathrm{v} / \mathrm{v})$ neutral buffered Formalin solution.

\subsection{4. $N F-\kappa B$ translocation into the nucleus}

To assess whether we were working within the inflammation period of IRI the translocation into the nucleus of the transcription factor NF$\kappa B$ (p65), a well-known regulator of inflammatory response genes, was assayed by Western blot. Nuclear extracts from the liver of control rats and rats that had underwent ischaemia-reperfusion were prepared by a modification of the method described in (de Oliveira-Marques et al., 2007). Briefly a homogenate was made in cytosolic lysis buffer $(10 \mathrm{mM}$ HEPES pH 7.6, 2 mM EDTA, $1.5 \mathrm{mM} \mathrm{MgCl}_{2}, 10 \mathrm{mM} \mathrm{KCl}$ and $300 \mathrm{mM}$ sucrose plus freshly added $2 \mathrm{mM}$ DTT, $0.1 \%$ Nonidet P-40 (v/v) and protease inhibitors: $1 \mathrm{mM}$ PMSF, $1.5 \mu \mathrm{g} / \mathrm{mL}$ benzamidin, $10 \mu \mathrm{g} / \mathrm{mL}$ leupeptin and $1 \mu \mathrm{g} / \mathrm{mL}$ pepstatin). After centrifugation at $270 \times g$ for $30 \mathrm{~s}$ the supernatant was collected and centrifuged at $1500 \times g$ for $3 \mathrm{~min}$ at $4{ }^{\circ} \mathrm{C}$. The pellet was washed once with the cytosolic lysis buffer and then resuspended with the nuclear lysis buffer (same as the cytosolic buffer except that $420 \mathrm{mM} \mathrm{NaCl}, 25 \%$ (v/v) glycerol, $0.1 \mathrm{mM}$ EGTA and $0.1 \mathrm{mM}$ EDTA were used, but neither sucrose nor Nonidet P40 were added to the buffer). The pellet was put on ice during $30 \mathrm{~min}$ and mixed by vortexing three times during this incubation. Nuclear proteins were collected after centrifugation at $10,000 \times g$ for $10 \mathrm{~min}$ at $4{ }^{\circ} \mathrm{C}$. Protein concentration was assayed using the Bradford method (Bradford, 1976).

Loading buffer [31.5 mM Tris- $\mathrm{HCl} \mathrm{pH} 6.8,10 \%$ (v/v) glycerol, 1\% $(\mathrm{w} / \mathrm{v})$ SDS, $0.005 \%(\mathrm{w} / \mathrm{v})$ bromophenol blue] was added to each sample of nuclear proteins that were then analysed by SDS-PAGE using a $10 \%(\mathrm{w} / \mathrm{v})$ polyacrylamide gel. After electrophoresis, proteins were transferred to a nitrocellulose membrane. The primary antibody for p65 (goat polyclonal anti-p65, 1:750, sc-372 from Santa Cruz Biotechnology, Santa Cruz, California, USA) was diluted in blocking solution (PBS, $0.1 \%$ Tween-20, 5\% milk) and incubated with the membrane for $1 \mathrm{~h}$ at room temperature. The membrane was then washed three times with washing solution (PBS with $0.1 \%(\mathrm{v} / \mathrm{v})$ Tween20) and incubated for $2 \mathrm{~h}$ at room temperature with peroxidase-conjugated anti-goat (sc-2020 from Santa Cruz Biotechnology, Santa Cruz, California, USA). Blots were developed using an ECL chemiluminescence system (Millipore). After detection of the labelled bands, images were acquired with ImageQuant LAS 500 (GE Healthcare) and protein expression was quantified with ImageJ. The expression of p65 was normalized using as loading control the intensity of protein bands (stained using Ponceau-S red) in a region of the membrane similar to the protein of interest as in (de Oliveira-Marques et al., 2007; OliveiraMarques et al., 2013).

\subsubsection{Assessment of liver ischaemia-reperfusion injury}

2.3.5.1. Haemogram and leukocyte count in blood. Blood counts of blood samples were performed on the ADVIA $^{\circledR} 120$ Hematology System (Siemens). The haemoglobin present in the samples was quantified at $546 \mathrm{~nm}$, after lysis, using a reagent containing $20 \mathrm{mM}$ potassium cyanide and $2 \%(\mathrm{w} / \mathrm{v})$ dimethyllaurylamine oxide (HGB reagent). The leukocyte count present in the samples was obtained using three specific reagents for the ADVIA $^{\circledast} 120$ System, including ADVIA ${ }^{\circledR} 120$ PEROX 1 ( $0.36 \mathrm{mmol} / \mathrm{L}$ sodium dodecyl sulfate, $620 \mathrm{mmol} / \mathrm{L}$ sorbitol, $8.35 \mathrm{mmol} / \mathrm{L} \mathrm{NaCl}, 5.5 \%$ (v/v) formaldehyde, $0.1 \mathrm{mmol} / \mathrm{L}$ Brij-35), ADVIA $^{\circledast} 120$ PEROX 2 (44.8 mmol/L 4-chloro-1-naphthol and $99.2 \%$ (v/v) diethylene glycol) and ADVIA ${ }^{\circledast} 120$ PEROX 3 (0.3\% (v/v) $\mathrm{H}_{2} \mathrm{O}_{2}$ and a stabilizer).

2.3.5.2. Enzymatic activities of liver injury biomarkers in serum. Aspartate aminotransferase (AST), alanine aminotransferase (ALT), alkaline phosphatase (ALP) and $\gamma$-glutamyl transferase $(\gamma$-GT) activities in serum were used as biomarkers of ischaemia-reperfusion injury. The enzyme activities were assayed using an ADVIA $^{\circledR}$ Chemistry System (Bayer HealthCare). AST was assayed according to Bergmeyer et al. (1978) and $1 \mathrm{U}$ is the quantity of AST that catalyzes the formation of $1 \mu \mathrm{mol}$ of $\mathrm{NAD}^{+}$per minute at $\mathrm{pH} 7.6$ and $37{ }^{\circ} \mathrm{C}$. ALT was determined according to Bergmeyer and Horder (1980) and $1 \mathrm{U}$ is the quantity of ALT that catalyzes the formation of $1 \mu \mathrm{mol}$ of $\mathrm{NAD}^{+}$per minute at pH 7.6 and $37^{\circ} \mathrm{C} . \gamma$-GT was assayed according to Shaw et al. (1977) and 
$1 \mathrm{U}$ is the quantity of $\gamma$-GT that catalyzes the formation $1 \mu \mathrm{mol}$ of 5 amino-2-nitro-benzoate per minute at $\mathrm{pH} 8.25$ and $30^{\circ} \mathrm{C}$. ALP was assayed according to Tietz et al. (1980) and $1 \mathrm{U}$ is the quantity of ALP that catalyzes the formation $1 \mu \mathrm{mol}$ of $p$-nitrophenol per minute at $\mathrm{pH} 10.3-10.4$ and $30^{\circ} \mathrm{C}$.

2.3.5.3. Histological studies. For histopathological examinations, livers were processed into paraffin blocks, sectioned at nominal $5 \mu \mathrm{m}$, mounted on glass microscope slides and stained with haematoxylin and eosin. The histological characterization consisted on the following parameters: presence of inflammation; sinusoidal congestion; portal congestion; centrilobular congestion; peripheral necrosis; infarctions; ductular changes and proliferation. This characterization is in accordance with the classification described by Suzuki et al. (1998).

2.3.5.4. Magnetic resonance microscopy. Magnetic resonance microscopy images were obtained at a $7 \mathrm{~T}$ magnetic field in a Bruker Avance III spectrometer equipped with a gradient magnetic field of $160 \mathrm{G} / \mathrm{cm}$, which allowed us the collection of Magnetic Resonance Imaging (MRI) with high spatial resolution. The organs were immersed in Fomblin for image acquisition.

MRM were obtained from $1 \mathrm{~mm}$ liver slices by a FLASH imaging procedure with an excitation angle of $30^{\circ}$, a Field of View of $3 \times 3 \mathrm{~cm}^{2}$, a $256 \times 256$ matrix and a repetition time in a range $200 \mathrm{~ms}$ to $320 \mathrm{~ms}$ and an echo-time of 6 to $10 \mathrm{~ms}$. The signal was evaluated 8 times for one image, and for each organ the echo-time was optimized to achieve the best contrast.

\subsection{Statistical analysis}

Results are given as the mean \pm standard deviation of at least 3 independent experiments made in triplicate, except where otherwise specified. For animal experiments, data is represented as the mean \pm standard deviation and statistical significance was tested using one-way ANOVA test. Comparison between each pair of groups was made using a two-tailed Student's $t$-test.

\section{Results and discussion}

\subsection{Characterization of SOD-enzymosomes and SOD-liposomes}

Two different liposomal formulations, SOD-enzymosomes i.e. SOD covalently linked to the distal end of DSPE-PEG long circulating liposomes and SOD-liposomes, i.e. PEG-liposomes with encapsulated SOD in the internal aqueous space were characterized in terms of their particle size, loading capacities and retention of the enzymatic activities. The results of the characterizations of the two formulations are shown in Table 1. Both formulations had the same mean size $(0.14-0.15 \mu \mathrm{m})$ with a low and equivalent polydispersity index. These two parameters are crucial to enable the comparison of the in vivo behaviour of the two formulations in terms of the long circulating properties and the capacity of accumulation in inflamed areas (Corvo et al., 1999, 2015; Martins et al., 2014). It should also to be highlighted that both formulations had approximately the same enzyme-to-lipid ratio $\left(\mathrm{SOD}_{\mathrm{e} / \mathrm{c}} /\right.$ lipid) thus enabling targeting the same dose of enzyme per carrier. SOD encapsulation efficiency for liposomes was about 2.5 fold lower than conjugation efficiency observed for enzymosomes which is due to the different technical protocols used to prepare the nanosystems, namely the extrusion procedure for sizing liposomes (Corvo et al., 2002). As proven earlier, the slight difference in terms of the enzyme activity observed in both formulations does not compromise the therapeutic outcomes when used in an arthritis model (Corvo et al., 2015).

\subsection{SOD-enzymosomes are more effective than SOD-liposomes in decreasing liver ischaemia-reperfusion injury}

To assess IRI and whether our liposomal formulations decreased the liver injury we used classical biochemical biomarkers of liver injury, haematological parameters, histological analysis and MRM.

\subsubsection{Enzymatic activities of liver injury biomarkers in serum}

Liver IRI can be assessed by determining the activities of several enzymes that are known biomarkers of liver cellular damage (AST, ALT, ALP and $\gamma$-GT). AST and ALT are known as being reliable markers for hepatocyte injury liver function leading to necrosis (Ozer et al., 2008). Damaged hepatocytes release their contents into the extracellular space, and so, increased levels of AST and ALT in serum indicate increased permeability and damage and/or necrosis of hepatocytes (Hsieh et al., 2015; Ozer et al., 2008). AST and ALT activities in serum have been previously found to be increased due to ischaemia-reperfusion injury (Gonzalez-Flecha et al., 1993). On the other hand, ALP and $\gamma$-GT, are both enzymes associated with the hepatocyte cell membrane, and their increased levels in the serum indicate hepatobiliary damage and cholestasis.

In Fig. 1 we can see that after $24 \mathrm{~h}$ reperfusion there was a 4 -fold increase in AST activity and a 3.3-fold increase in ALT activity in rat serum, while there were more modest increases of ALP and $\gamma$-GT activities (a 1.3-fold for ALP and 1.5- fold for $\gamma$-GT activity). The increased activity of all these enzymes in rat serum after indicates that IR led to liver IRI. In contrast SHAM-operated rats showed no changes in all enzymatic activity levels when compared to control rats (Fig. 1).

All three SOD-treatments used were effective in returning ALP activity to SHAM/Control levels, unlike the other biomarkers where there were differences in the efficacy of the SOD treatments. Treatment with both free SOD and SOD-liposomes had no effect in decreasing the higher AST and $\gamma$-GT activities induced by IR, but decreased ALT activity although not to SHAM levels. By contrast, upon treatment with SOD-enzymosomes all enzymatic activities decreased more than with the other SOD formulations with ALT, ALP and $\gamma$-GT showing no difference from activity levels found in SHAM rats. Results obtained for AST agree with our previous studies using SOD liposomal formulations for liver ischaemia-reperfusion (Corvo et al., 2015).

Overall, when comparing SOD-enzymosomes with SOD-liposomes the former were more effective in reducing levels of AST, ALT and $\gamma$-GT activities after ischaemia reperfusion. So, results with biochemical liver injury biomarkers suggest that SOD-enzymosomes are the more effective formulation for decreasing IRI.

Table 1

Characteristics of SOD-enzymosomes and SOD-liposomes used for the in vivo studies

\begin{tabular}{|c|c|c|c|c|c|}
\hline Formulation & Particle size $(\mu \mathrm{m})$ & PdI & $\mathrm{SOD}_{\mathrm{e} / \mathrm{c}} / \mathrm{lipid}(\mu \mathrm{g} / \mu \mathrm{mol})$ & E(/C).E. (\%) & Ret. Enz. act. (\%) \\
\hline SOD-liposomes & $0.14 \pm 0.02$ & 0.070 & $14.4 \pm 0.5$ & $9 \pm 2$ & $>85$ \\
\hline SOD-enzymosomes & $0.15 \pm 0.02$ & 0.178 & $12.5 \pm 0.9$ & $22 \pm 2$ & $60-70$ \\
\hline
\end{tabular}

$\mathrm{SOD}_{\mathrm{e} / \mathrm{c}}$ /lipid - encapsulated or conjugated SOD/lipid; E(/C).E. - encapsulated or conjugated efficiency; particle size - Z-average, PdI - polydispersity index.

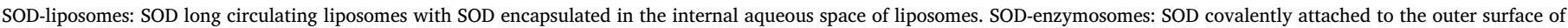
long circulating liposomes (PEG end). 

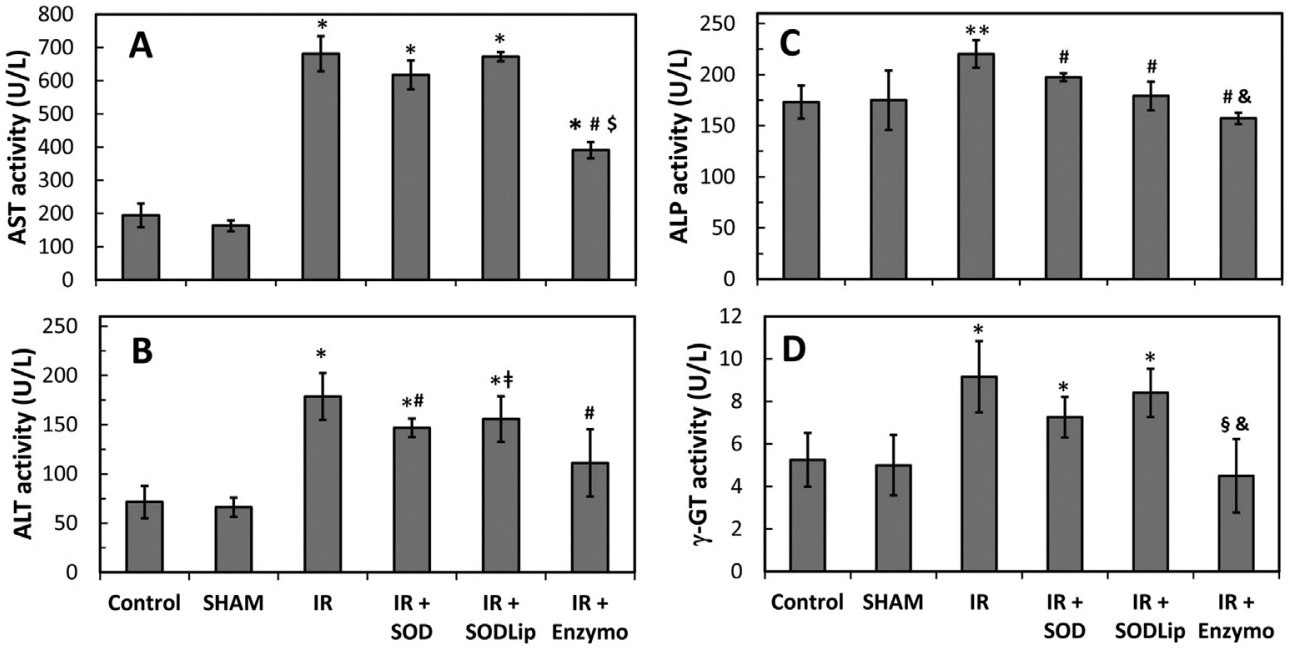

Fig. 1. Activities of several enzymatic biomarkers of liver ischaemia-reperfusion injury. Activities of (A) aspartate aminotransferase (AST), (B) alanine aminotransferase (ALT), (C) alkaline phosphatase (ALP) and (D) $\gamma$-glutamyl transferase $(\gamma$-GT) were determined in rat serum as described in materials and methods. Control, rats with no ischaemia-reperfusion (IR) induction and no treatment; SHAM, rats where ischaemia/reperfusion induction was simulated; IR, rats which underwent ischaemia/ reperfusion and with no treatment. Rats that underwent ischaemia-reperfusion were injected with either free SOD (IR + SOD), SOD-liposomes (IR + SODLip), and with SOD-enzymosomes (IR + Enzymo). Values are the mean \pm standard deviation for $\mathrm{n}>(3-10)$ animals per group. $* \mathrm{P}<0.01$ vs SHAM; $* * \mathrm{P}<0.05$ vs SHAM; ${ }^{\#} \mathrm{P}<0.01$ vs IR; ${ }^{~} \mathrm{P}<0.05$ vs IR; ${ }^{\S} \mathrm{P}<0.05$ vs SOD; ${ }^{\text {}} \mathrm{P}<0.01$ vs IR + SODLip; ${ }^{\&} \mathrm{P}<0.05$ vs IR + SODLip.

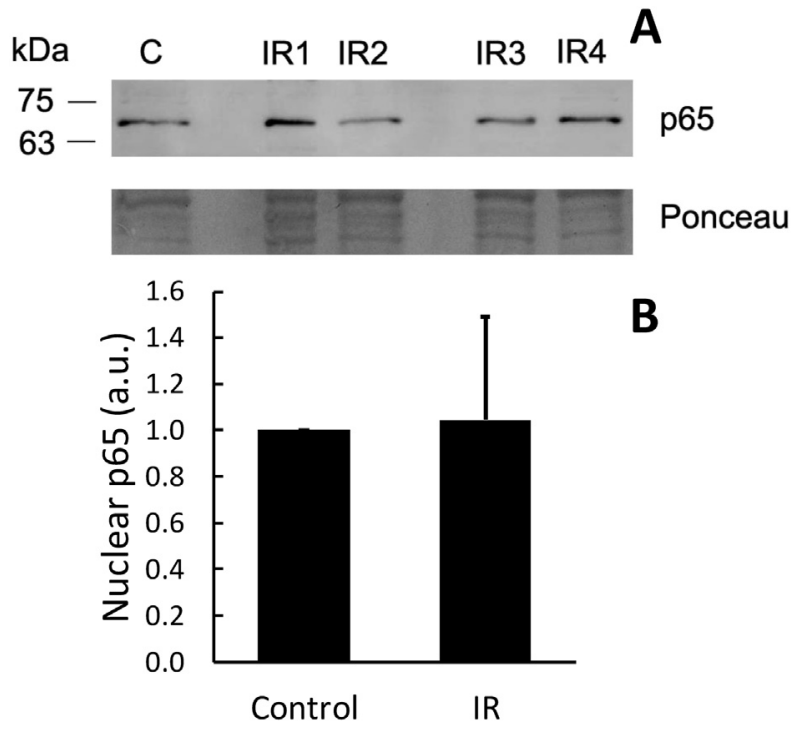

Fig. 2. Liver ischaemia-reperfusion does not lead to increased p65 translocation into the nucleus. The levels of nuclear p65 were followed by western blot. (A) Representative immunoblots of $n=4$ independent experiments showing the levels of p65 in nuclear extracts from livers of control rats $(\mathrm{C})$ and of rats who had undergone ischaemia-reperfusion (IR) after $24 \mathrm{~h}$. (B) Signal intensity quantification of protein levels expressed as the mean \pm standard deviation in arbitrary units (a.u.) relative to control rats. Protein levels were normalized to the protein loading using the intensity of protein bands (stained using Ponceau-S red) in a region of the membrane similar to the protein of interest.

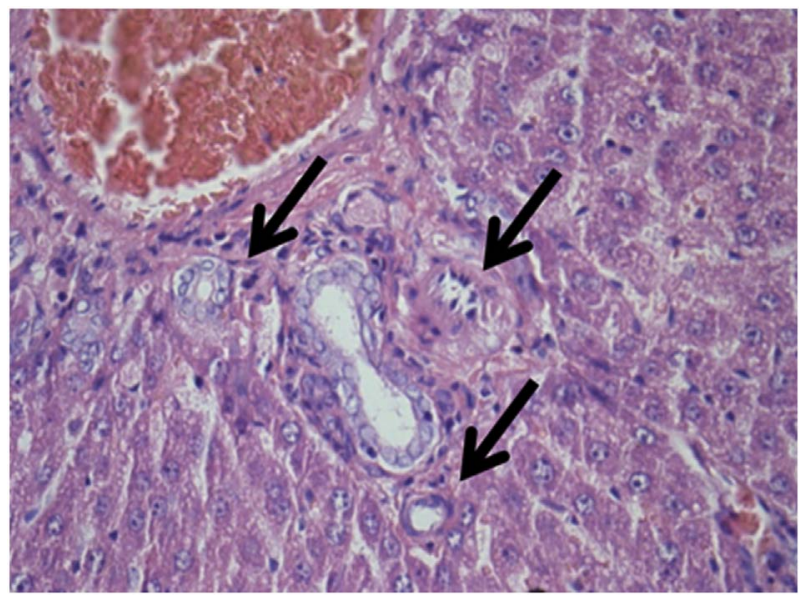

Fig. 3. Rat liver ischaemia reperfusion induces marginal ductular proliferation. Representative light microphotograph of hepatic sections stained with haematoxylin and eosin. The arrows indicate a newly formed duct.

\subsubsection{Inflammatory response and liver histological studies}

Analysis of blood samples from the different groups of animals used in the study was performed to obtain the leukocyte count to determine the state of the animals' immune system and, also, the amount of haemoglobin. Haemoglobin values $(12-15 \mathrm{~g} / \mathrm{dL})$ were within the normal range for all groups and no differences were observed between groups. This suggests that neither the surgery nor the administration of the two SOD liposomal formulations (SOD-Liposomes and SOD-enzymosomes) and of "free" SOD, caused anaemia.

Inverted neutrophil-lymphocyte ratios, i.e. when the percentage of

Table 2

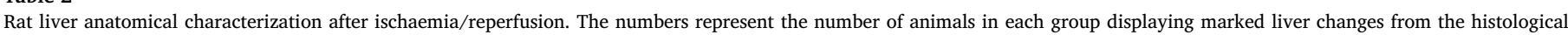
analysis.

\begin{tabular}{|c|c|c|c|c|c|c|}
\hline \multirow[b]{2}{*}{ Rat treatment group } & \multirow[b]{2}{*}{ Inflammation } & \multicolumn{5}{|c|}{ Number of animals affected } \\
\hline & & Sinusoidal congestion & Portal congestion & Centrilobular congestion & Peripheral necrosis & Ductular changes \\
\hline Control/SHAM $(\mathrm{n}=1)$ & 0 & 0 & 0 & 0 & 0 & 0 \\
\hline $\operatorname{IR}(n=5)$ & 1 & 4 & 4 & 4 & 4 & 4 \\
\hline $\mathrm{IR}+\mathrm{SOD}(\mathrm{n}=3)$ & 0 & 3 & 3 & 3 & 3 & 0 \\
\hline IR + SODLip $(n=4)$ & 1 & 1 & 3 & 3 & 2 & 0 \\
\hline IRI + Enzymo $(n=4)$ & 1 & 2 & 1 & 2 & 0 & 1 \\
\hline
\end{tabular}

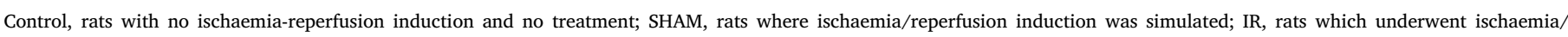

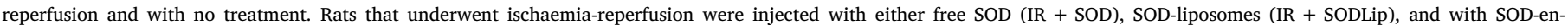
zymosomes (IR + Enzymo). 

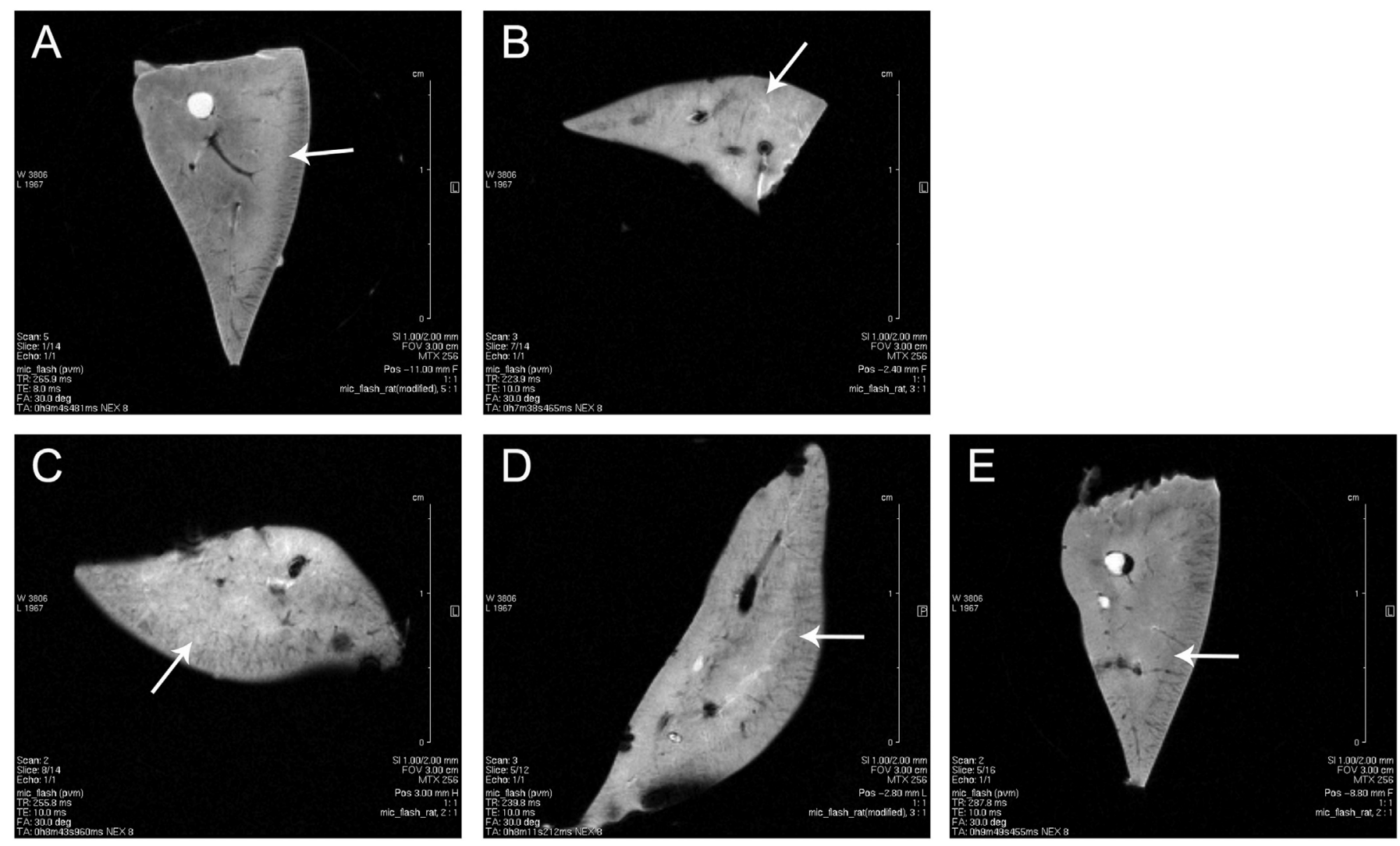

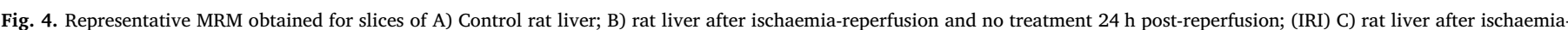

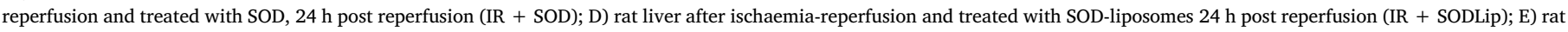
liver after ischaemia-reperfusion and treated with SOD-enzymossomes (IR + Enzymo), $24 \mathrm{~h}$ post reperfusion.

neutrophils is higher than the percentage of lymphocytes, may indicate that an inflammation is occurring and are also associated with physiological response to situations of any kind of stress or change (Simões et al., 2005). As expected, animals of the control and sham-operated (SHAM) groups, did not display inverted neutrophil-lymphocyte ratios. In all the 4 operated non-treated and treated animal groups an inverted neutrophil-lymphocyte ratio occurred in less than $30 \%$ of the animals but there was no correlation with the treatment. We also analysed by Western blot the nuclear levels of NF-kB (p65), a well-known transcriptional regulator of inflammatory response genes, in control and operated animals (Fig. 2). We found no significant differences in the nuclear levels of NF-KB (p65) between both groups but the high variability in the levels of nuclear p65 from IR liver extracts, suggests that inflammatory events may be happening in the liver of some of the animals analysed. This is in agreement with the inverted neutrophillymphocyte ratio found in some animals after IR. Since no significant changes were found in the levels of nuclear p65 after IR when compared to control rats we did not further analyse p65 nuclear levels in the liver of treated groups.

Rat liver from controls and SOD treated groups were analysed histologically and typical parameters used to assess liver injury such as inflammation, sinusoidal, portal and centrilobular congestion, peripheral necrosis and ductular changes were determined (see Table 2). In animals that underwent IR, all analysed parameters were present in the majority of animals indicating that there was IRI after $24 \mathrm{~h}$. In all groups inflammation was a rare event which was in accordance to results observed for p65 nuclear levels and the leucocyte profile inversion. The number of incidences of sinusoidal congestion, normally a consequence of venous outflow impairment, decreased with all SOD treatments but the decrease was more relevant for the animals treated with SOD-liposomes and SOD-enzymosomes. SOD-enzymosomes were the more effective therapeutic approach to decrease portal and centrilobular congestion or peripheral necrosis observed in the liver after IR. These necrotic or inflammatory changes may be linked to the short ischaemia period (30 min). However, the greatest distinguishing feature of liver IRI found in the histological studies consisted of ductular cell proliferation (see Fig. 3). Ductular cell proliferation in the liver after IR was prevented with all three SOD treatments used. These ductular changes did not occur in larger biliary pathways and are commonly related to degenerative modifications in small biliary canaliculi, which are small structures consisting of 5 to 6 endothelial cells mostly present at the periphery, that upon endothelial cell death can obstruct the canalicular lumen. So, it can be assumed that vascular congestion is a common and reversible histopathological occurrence in liver after IR, but preventive antioxidant therapy, such as administration of free SOD and SOD-liposomes or SOD-enzymosomes, can prevent injury to these small ductular structures.

Although our study was not clinically oriented, it is worth noticing that the disappearance of peripheral small biliary structures leading to a so-called, peripheral ductopenia, after LT or ischaemia-related biliary disease, is a major clinical issue in LT, affecting graft quality and post LT course. Therefore, since our results showed that SOD liposomal formulations are useful to prevent ductular damage, the use of SOD liposomal formulations aimed at preventing a situation that can compromise the graft survival may deserve further investigation. Overall, taking into account all the parameters used for rat liver anatomical characterization to determine IRI, of the three formulations used SODenzymosomes seemed to be therapeutically more efficient than SODliposomes, with SOD being the less effective. These results are in agreement with the biochemical studies where SOD-enzymosomes showed a better therapeutic effect than SOD-liposomes. 


\subsection{Liver IRI can be followed by magnetic resonance micro-images}

MRM imaging at a $7 \mathrm{~T}$ magnetic field was obtained from slices images of the liver from control rats and from rats who underwent ischaemia reperfusion without or with treatment with liposomal SOD and SOD formulations $24 \mathrm{~h}$ post-reperfusion (Fig. 4). As can be seen in Fig. 4B, a high intensity signal at the periphery, as well as vasodilatation was observed in liver of rats subjected to IR when compared to liver of control rats (Fig. 4A). This highlighted that in IRI the peripheral tissue was more affected, losing its peripheral delimitation and displaying dilated vascular structure, than central parts of the liver. This is in agreement with previous results obtained with MRM where liver tissue after IR displayed a disrupted vascular architecture when compared to normal liver, with the disrupted vascular architecture occurring more at the peripheral tissue than at the central tissue (Martins et al., 2014). By the observation of Fig. 4C, it can be concluded that treatment with free SOD (IR + SOD) had no effect in the affected peripheral tissue found after IR. However, protection against IRI occurred for both liposomal treatments (IR + SODLip, IR + Enzymo), as it could be seen that the liver displayed a different vasculature pattern with a better preservation of the liver peripheral structure (displaying peripheral delimitation and no vascular vasodilatation) (Fig. 4D and E). Moreover, the liver of animals treated with SOD-enzymosomes displayed a more intense and homogeneous signal (Fig. 4E), when compared to the liver of IR rats (Fig. 4B).

Also, results obtained by MRM agree with the activities of enzymatic biomarkers of liver cellular damage (Fig. 2) and with the histopathological changes (Table 2 and Fig. 3) that seem to be the hallmark of liver IRI i.e. vascular congestion, peripheral necrosis and ductular changes. MRM images may essentially reflect the vascular congestion, also found in histopathology, but do not allow to distinguish it from injury to small biliary peripheral structures. Overall, results from MRM images show that both liposomal formulations were able to attenuate IRI but that SOD-enzymosomes were more effective, which is in agreement with the results obtained both with the biochemical markers and with the histological analysis.

\section{Conclusions}

Biochemical biomarkers of liver injury and liver histology showed that IRI occurs in our model of liver ischaemia reperfusion. MRM at $7 \mathrm{~T}$ was used to monitor IRI and effects seen on MRM images correlated with the results from classical biochemical biomarkers of liver injury and liver histology. Furthermore, MRM at $7 \mathrm{~T}$ images allowed confirming that the damage to the liver tissue was mostly peripheral with biliary ductular damage and a disrupted vascular architecture as main consequences of IRI. Thus, we were able to show that MRM at $7 \mathrm{~T}$ is a useful technique to follow IRI. Also, MRM allowed to distinguish the therapeutic effects of both liposomal formulations used. Both SOD liposomal formulations used could attenuate liver IRI. However, SODenzymosomes, where SOD is at the liposomal surface, were more effective in reducing IRI as shown by results obtained for biochemical markers, histology and MRM and this may be due to a short therapeutic window in liver IRI. This work confirms that the use of SOD liposomal formulations, particularly SOD-enzymosomes, for decreasing liver IRI in a rat liver model of ischaemia reperfusion is a useful therapeutic approach.

\section{Acknowledgements}

We wish to acknowledge Dr. Luísa Cyrne for the help on the NF-kB translocation assay and Instituto de Investigação Bento da Rocha Cabral for free animal supply (ischaemia/reperfusion model).

This work was supported by Fundação para a Ciência e a Tecnologia, Portugal (grants: PEst-OE/QUI/UI0612/2013; PEst-OE/ UID/DTP/04138/2013); Sociedade Portuguesa de Transplantação
(Grant Bolsa de Investigação SPT/Astellas Farma, 2010).

\section{References}

Beckman, J.S., Koppenol, W.H., 1996. Nitric oxide, superoxide, and peroxynitrite: the good, the bad, and ugly. Am. J. Phys. 271, C1424-C1437.

Bergmeyer, H.U., Horder, M., 1980. International federation of clinical chemistry. Scientific committee. Expert panel on enzymes. IFCC document stage 2, draft 1; 197911-19 with a view to an IFCC recommendation. IFCC methods for the measurement of catalytic concentration of enzymes. Part 3. IFCC method for alanine aminotransferase. J. Clin. Chem. Clin. Biochem. 18, 521-534.

Bergmeyer, H.U., Scheibe, P., Wahlefeld, A.W., 1978. Optimization of methods for aspartate aminotransferase and alanine aminotransferase. Clin. Chem. 24, 58-73.

Bradford, M.M., 1976. A rapid and sensitive method for the quantitation of microgram quantities of protein utilizing the principle of protein - dye binding. Anal. Biochem. $72,248-254$.

Carillon, J., Rouanet, J.M., Cristol, J.P., Brion, R., 2013. Superoxide dismutase administration, a potential therapy against oxidative stress related diseases: several routes of supplementation and proposal of an original mechanism of action. Pharm. Res. 30, 2718-2728.

Cheung, J.S., Wang, X., Zhe, S.P., 2011. Magnetic resonance characterization of ischemic tissue metabolism. Open Neuroimaging J. 5, 66-73.

Corvo, M.L., Boerman, O.C., Oyen, W.J., van Bloois, L., Cruz, M.E.M., Crommelin, D.J.A., Storm, G., 1999. Intravenous administration of superoxide dismutase entrapped in long circulating liposomes. II. In vivo fate in a rat model of adjuvant arthritis. Biochim. Biophys. Acta 1419, 325-334.

Corvo, M.L., Jorge, J.C.S., van't Hof, R., Cruz, M.E.M., Crommelin, D.J.A., Storm, G., 2002. Superoxide dismutase entrapped in long-circulating liposomes: formulation design and therapeutic activity in rat adjuvant arthritis. Biochim. Biophys. Acta 1564, 227-236.

Corvo, M.L., Marinho, H.S., Marcelino, P., Lopes, R.M., Vale, C.A., Marques, C.R., Martins, L.C., Laverman, P., Storm, G., Martins, M.B., 2015. Superoxide dismutase enzymosomes: carrier capacity optimization, in vivo behaviour and therapeutic activity. Pharm. Res. 32, 91-102.

Corvo, M.L., Marinho, H.S., Martins, M.B.F., 2016. Nanomedicines as a strategy for the therapeutic use of superoxide dismutase. In: Phillips, N.H. (Ed.), Superoxide Dismutase (SOD): Sources, Therapeutics and Health Benefits. Nova Science Publisher's, Inc. - Nova Biomedical, New York, pp. 135-170.

Diesen, D.L., Kuo, P.C., 2011. Nitric oxide and redox regulation in the liver: part II. Redox biology in pathologic hepatocytes and implications for intervention. J. Surg. Res. 167, 96-112.

Driehuys, B., Nouls, J., Badea, A., Bucholz, E., Ghaghada, K., Petiet, A., Hedlund, L.W., 2008. Small animal imaging with magnetic resonance microscopy. ILAR J. 49, 35-53.

Gonzalez-Flecha, B., Cutrin, J.C., Boveris, A., 1993. Time course and mechanism of oxidative stress and tissue damage in rat liver subjected to in vivo ischemia-reperfusion. J. Clin. Invest. 91, 456-464.

Hsieh, Y.L., Yeh, Y.H., Lee, Y.T., Huang, C.Y., 2015. Protective effects of Cholestin on ethanol induced oxidative stress in rats. J. Sci. Food Agric. 95, 799-808.

Jaeschke, H., 2011. Reactive oxygen and mechanisms of inflammatory liver injury: present concepts. J. Gastroenterol. Hepatol. 26, 173-179.

Jaeschke, H., Farhood, A., 2002. Kupffer cell activation after no-flow ischemia versus hemorrhagic shock. Free Radic. Biol. Med. 33, 210-219.

Klune, J.R., Tsung, A., 2010. Molecular biology of liver ischemia/reperfusion injury: established mechanisms and recent advancements. Surg. Clin. North Am. 90, 665-677.

Lowry, O.H., Rosebrough, N.J., Farr, A.L., Randall, R.J., 1951. Protein measurement with the folin phenol reagent. J. Biol. Chem. 193, 265-275.

Lutz, J., Thurmel, K., Heemann, U., 2010. Anti-inflammatory treatment strategies for ischemia/reperfusion injury in transplantation. J. Inflamm. (Lond.) 7.

Martins, M.B.F., Corvo, M.L., Marcelino, P., Marinho, H.S., Feio, G., Carvalho, A., 2014. New long circulating magnetoliposomes as contrast agents for detection of ischemiareperfusion injuries by MRI. Nanomedicine: NBM 10, 207-214.

Misra, H.P., Fridovich, I., 1972. The role of superoxide anion in the autoxidation of epinephrine and simple assay for superoxide dismutase. J. Biol. Chem. 247, 3170-3175.

de Oliveira-Marques, V., Cyrne, L., Marinho, H.S., Antunes, F., 2007. A quantitative study of NF-kappa B activation by $\mathrm{H} 2 \mathrm{O} 2$ : relevance in inflammation and synergy with TNFalpha. J. Immunol. 178, 3893-3902.

Oliveira-Marques, V., Silva, T., Cunha, F., Covas, G., Marinho, H.S., Antunes, F., Cyrne, L., 2013. A quantitative study of the cell-type specific modulation of c-Rel by hydrogen peroxide and TNF-alpha. Redox Biol. 1, 347-352.

Ozer, J., Ratner, M., Shaw, M., Bailey, W., Schomaker, S., 2008. The current state of serum biomarkers of hepatotoxicity. Toxicology 245, 194-205.

Rouser, G., Fleischer, S., Yamamoto, A., 1970. Two dimensional thin layer chromatographic separation of polar lipids and determination of phospholipids by phosphorus analysis of spots. Lipids 5, 494-496.

Serracino-Inglott, F., Habib, N.A., Mathie, R.T., 2001. Hepatic ischemia-reperfusion injury. Am. J. Surg. 181, 160-166.

Shaw, L.M., London, J.W., Fetterolf, D., Garfinkel, D., 1977. Gamma-glutamyltransferase kinetic-properties and assay conditions when gamma-glutamyl-4-nitroanilide and its 3-carboxy derivative are used as donor substrates. Clin. Chem. 23, 79-85.

Simões, S.I.D., Delgado, T.C., Lopes, R.M., Jesus, S., Ferreira, A.A., Morais, J.A., Cruz, M.E.M., Corvo, M.L., Martins, M.B.F., 2005. Developments in the rat adjuvant arthritis model and its use in therapeutic evaluation of novel non-invasive treatment by SOD in Transfersomes. J. Control. Release 103, 419-434.

Suzuki, S., Nakamura, S., Sakaguchi, T., Mitsuoka, H., Tsuchiya, Y., Kojima, Y., Konno, H., 
Baba, S., 1998. Pathophysiological appraisal of a rat model of total hepatic ischemia with an extracorporeal portosystemic shunt. J. Surg. Res. 80, 22-27.

Tietz, N.W., Burtis, C., Ervin, K., Petitclerc, C.J., Rinker, A.D., Zygowicz, E., 1980.

Progress in the development of a recommended method for alkaline-phosphatase activity measurements. Clin. Chem. 26, 1023.
Wang, C.-H., Smith, R.L., 1975. Lowry determination of protein in the presence of triton X-100. Anal. Biochem. 63, 414-417.

Zhai, Y., Petrowsky, H., Hong, J.C., Busuttil, R.W., Kupiec-Weglinski, J.W., 2013.

Ischaemia-reperfusion injury in liver transplantation-from bench to bedside. Nat. Rev. Gastroenterol. Hepatol. 10, 79-89. 